\title{
Dynamics of complex coordination during disease outbreak
}

\author{
F. Bdeir ${ }^{1}$, L. Hossain ${ }^{1}$, J. Crawford ${ }^{2}$, C. Kewely ${ }^{3} \&$ J. Carter $^{3}$ \\ ${ }^{I}$ Project Management Graduate Program, \\ The University of Sydney, Australia \\ ${ }^{2}$ Faculty of Agriculture, Food and Natural Resources, \\ The University of Sydney, Australia \\ ${ }^{3}$ Hunter New England Health Network Services, Australia
}

\begin{abstract}
When multiple agencies respond to a disease outbreak (i.e., H1N1 and SARS), the coordination of actions is complex and evolves over time. We know little about this because there has been no systematic empirical study of the dynamics of coordination during a disease outbreak. In this paper, we introduce an approach to the analysis of multi-agency intervention during a disease outbreak using methods previously applied to the study of social networks. The discussion is supported by a qualitative study of the implementation of the results of the analysis. We reveal that profound understanding of social network behaviour and emerging coordination concepts are corner stones for successful outbreak intervention. We look qualitatively at how Hunter New England Area Health Services applied these concepts to lead a successful coordination plan during an H1N109 endemic.
\end{abstract}

Keywords: disease outbreak coordination, complex coordination, network analysis, disaster management, coordination.

\section{Introduction}

Disaster management is inherently complex due to the interdependent nature of the responses from multiple organizations that have responsibility for dealing with the situation collectively (Comfort et al. [1]). It has long been advocated that standard hierarchal management structures may not necessarily be the most efficient ones in such situations (Neal and Phillips [2]). This is due to the 
demanding requirement for extensive inter-organizational interactions, communication, and collaboration with interwoven dependencies (Chen et al. [3]). Disease outbreak can be considered as a unique type of disaster where the dynamics of the situation is particularly important. It is distinct from earthquakes, bushfire or floods that can only affect a particular geographical area Table 1 below is a comparison chart highlighting some differences between disease outbreak and bushfire or flood disasters:

Table 1: Differences between disease outbreak and bushfire/flood disasters.

\begin{tabular}{|l|l|}
\hline Disease outbreak & Bushfire/flood \\
\hline $\begin{array}{l}\text { Wave pattern behaviour determined } \\
\text { by the pathogen infectivity; might re- } \\
\text { infect the same affected } \\
\text { area/population. }\end{array}$ & $\begin{array}{l}\text { After burning a certain area, does not } \\
\text { return to it. }\end{array}$ \\
\hline $\begin{array}{l}\text { Outbreaks can cross geographic } \\
\text { boundaries to be a global } \\
\text { phenomenon (H1N109, SARS) }\end{array}$ & $\begin{array}{l}\text { Bounded by geographical } \\
\text { characteristics (Bush/rivers } \\
\text { locations). }\end{array}$ \\
\hline $\begin{array}{l}\text { Population discriminative; usually } \\
\text { some population segments (elderly, } \\
\text { children) are more vulnerable than } \\
\text { others. }\end{array}$ & $\begin{array}{l}\text { Population is targeted based on } \\
\text { geographical location vulnerability } \\
\text { alone. }\end{array}$ \\
\hline $\begin{array}{l}\text { Mutative and adaptive (influenza is } \\
\text { the best example). This antigenic-drift } \\
\text { adds to the complexity of the spread } \\
\text { patterns. }\end{array}$ & N/A \\
\hline $\begin{array}{l}\text { The spread patterns are influenced by } \\
\text { different factors including pathogen } \\
\text { contagion, demography and } \\
\text { behaviour }\end{array}$ & $\begin{array}{l}\text { Spread is based on simple factor(s) } \\
\text { mainly geographical characteristics. }\end{array}$ \\
\hline $\begin{array}{l}\text { Can be nature made or man-made } \\
\text { (bio-terrorism) }\end{array}$ & $\begin{array}{l}\text { Can be nature made or man-made } \\
\text { (arsonists) }\end{array}$ \\
\hline
\end{tabular}

A considerable amount of research has been undertaken into disease outbreaks from an epidemiological perspective including the role of pathogen transformation, mutation and infection (Fraser et al.[4]) and the modelling of disease spread (Newman [5]). However, there has been relatively little work been done on modelling the response of the multiple agencies responsible for dealing with the outbreak (Chen et al. [3]). The following reported cases of H1N109 outbreak in Australia, New South Wales state (NSW) (Eastwood et al. [6]):

Table 2 shows that Hunter New England (HNE) had the lowest transmission rate ( 0.9 per 100000 population), whereas an adjacent health area (North Coast) had a transmission rate more than twice as large (2 per 100 000). Also, HNE 
Table 2: Confirmed H1N109 infection rates in the eight New South Wales area health services at the end of the contain phase, 17 June 2009.

\begin{tabular}{|c|c|c|c|}
\hline $\begin{array}{l}\text { New South Wales area health } \\
\text { service }\end{array}$ & Population $^{\dagger}$ & Confirmed cases & Rate per 100000 \\
\hline \multicolumn{4}{|l|}{ Rural } \\
\hline Hunter New England & 862967 & 8 & 0.9 \\
\hline Greater Southern & 483282 & 42 & 8.7 \\
\hline Greater Western & 301052 & 9 & 3.0 \\
\hline North Coast & 495329 & 10 & 2.0 \\
\hline \multicolumn{4}{|l|}{ Metropolitan } \\
\hline $\begin{array}{l}\text { Northern Sydney/Central } \\
\text { Coast }\end{array}$ & 1134200 & 33 & 2.9 \\
\hline $\begin{array}{l}\text { South Eastern Sydney } \\
\text { Illawarra }\end{array}$ & 1209111 & 46 & 3.8 \\
\hline Sydney South West & 1394652 & 82 & 5.9 \\
\hline Sydney West & 1131294 & 83 & 7.3 \\
\hline New South Wales total' & 7011886 & 313 & 4.5 \\
\hline
\end{tabular}

tPopulation figures are based on estimated residential population 31 December 2008

TThe discrepancy with the NSW total in Table 1 is due to differences in population projections.

had the lowest number of confirmed cases in NSW state. Taking into consideration that demographics are similar and pathogen is the same, then management and coordination of the response to the outbreak is one factor affecting the infection rate. This effort is led by the corresponding agencies in each individual state and area health services within the states.

Successful intervention is a direct consequence of successful coordination. It is the coordination effort that brings together different types of resources such as information, expertise, and supplies (Chandler [7]) for the most efficient intervention plan. In this paper, we use measures from social network theory to better understand the dynamics of inter-organisational coordination during disease outbreaks. We collect disease outbreak coordination data from Hunter New England (HNE) area health services in New South Wales, Australia for demonstrating the effectiveness of these network-based measures to accomplish an effective coordination and communication plan that will contribute to the reduction of infected cases and transmission rates.

\section{Background}

In this section, we introduce the necessary theoretical background relating to coordination, coordination complexity in disasters, and social network analysis.

Coordination is increasingly seen important as organisations become more reliant on interdisciplinary teams of specialties and distributed operations for addressing complicated situations demanding a multi organisational response (Faraj and Xiao [8]). Malone defined coordination as "the act of managing interdependencies between activities performed to achieve a goal" (Malone and Crowston [9]). 


\subsection{Coordination complexity}

In multiagency dynamic environments during extreme events has been studied by Kapucu [10] using a framework primarily drawn from dynamic network theory and complex adaptive systems theory. Kapucu observed that coordination in extreme events is guided by a group of interconnected actors who necessarily rely on each other to achieve the goals collectively.

\subsection{Coordination and communication in disasters}

A major facet of coordination is communication (Feczak [11]). A study by Miller and Moser [12] suggests that "Communication can play a key role in the ability of agents to reach, and maintain, superior coordination". The two concepts are linked because communication can be regarded as a necessary and sufficient precedent associated with coordination. The coordination structure for disease outbreaks cannot be modelled or analysed using current standard and static coordination methods that focus on market theory proposed by Malone [9]. The concept of dynamic emerging coordination (Comfort et al. [1]) is seen to be better suited to model the inter-organisational communication where agencies have a tendency to establish, drop, and enhance communication links over time in order to achieve the optimal coordination scheme. We propose that emerging coordination can be best modelled as a complex adaptive system. Some of the characteristics of such coordination are:

\subsubsection{Robust information flow}

Information sharing can be regarded as the backbone of any successful coordination since the essential situational data necessary for quick adaptation (Iannella and Henricksen [13]).

\subsubsection{Environment dependency}

The coordination context is a direct result of the environment motivating the coordination. Besides dictating the resources sought after, the environment also influences the nature of the organisations that coordinate with each other's.

\subsubsection{Self-organising behaviour}

Self-organisation is a dynamic and adaptive process where systems acquire and maintain structure without external control (De Wolf and Holvoet [14]). Under these circumstances, operations will autonomously organise themselves within the whole coordination structure.

\section{Theory of networks and coordination}

Visualising inter-organisational coordination as a network structure is not uncommon. Hossain [15] has shown the viability of using network modelling for inter-organisational coordination and collaboration. Using this modelling approach, a node represents an entity (organisation, agent), and the links represent communication channels. Modelling emergent coordination as a 
network structure can be beneficial as it can incorporate the dynamism of the process of emergence. Below are some of the measures that can be applied in such a coordination structure.

\subsection{Degree centrality}

Centrality is the number of links to and from an organisation measured within the locality of the specific node (Freeman [16]). There can be in-degree centrality being the number of ties received indicating the importance of this node for its connected neighbours. And out-degree as the numbers of outbound links indicates that the organisation is in need of resources.

\subsection{Betweenness centrality}

It is a measure of the nodes that lie on the shortest path that connects everyone else in the network (Freeman [16]). This means that these nodes have quick access to new information and share this information with others.

\subsection{Weak ties}

Weak ties are efficient in knowledge sharing (Granovetter [17]). It provides access to new information since they bridge otherwise disconnected nodes or groups (or what is known as structural holes) (Hansen [18]). A bridge is a line in a network which provides the only path between structural holes

One other facet of coordination and networks is the informal coordination. It can be defined as when individuals or organisations establish communication networks outside the standard coordination structure to "get things done" (Han [19]). Informal networks can fill the lines that formal channels ignore, or capitalize existing ones to circumvent their complications, inefficiencies or even their inaccuracies. Informal networks are an integral part of any coordination process. Indeed, Cross et al. [20] even stated that, "work increasingly occurs through informal networks of relationships rather through channels tightly prescribed by formal reporting structure of detailed work processes" (Cross et al. [20]).

In a multi-organisational coordination, it is expected that organisations will efficiently utilise their existing links or establish new ones to maximise their fit and access to required resources.

\section{$4 \quad$ H1N109 virus case study}

Since there are not many studies that deal with organisational dynamics at play in the disease outbreak context, we use an investigative approach with a qualitative case study to capture rich information from senior health disaster management practitioners in the field. This provides a better insight into the complexity of the problem, a better understanding of the context, and a much needed holistic view of the entire coordination system. 
Our case study examines the coordination scenario that took place in 2009 when the H1N109 virus endemic was declared by WHO. Australia had its first confirmed swine flu case in Brisbane on 7 May 2009 (Eastwood et al. [6]). Worldwide WHO figures reported 4.4 fold case increases during June 2009 in confirmed cases whereas in Australia there was 13.4 fold case increases for the same period. The higher Australian rate can be partially attributed to the coinciding influenza season in southern winter (Eastwood et al. [6]).

In this case study, we explore the multi-agency coordination and communication that took place in Hunter New England Area Health Services (HNEAHS) during the endemic. HNEAHS is located in northern NSW within a geographical area of over 130,000 square kilometres, spans 25 local council areas, and has a population of about 870,000. HNEAHS activates the Health Service Functional Area Coordination (HSFAC) centre during major health crises. HSFAC is responsible for high level response operations, providing intelligence and guidance, and monitoring the cases reported by the "Front Line" (i.e., ED, GPs and other relevant health professionals). HSFAC objectives are as follows:

- $\quad$ Send a unified message to the community.

- Have standardized information flow to the front line (i.e., EDs, GPs).

- Apply robust "case definition" communication policy between receiving it from the state level until deployed in the front line.

- Providing intelligence and relevant information to the front line

- Keep the different sectors of the industry informed about the outbreak.

The interview data used here was collected on October 2010. We conducted semi-structured interviews with the senior HSFAC leaders in HNE. The interviews focused on recalling the communication and coordination processes that took place during the H1N109 endemic. These exploratory interviews were structured in to five sections where each section focused on one aspect of the coordination process. The sections and their relative primary questions are presented in table 3 below:

Table 3: Summarises the main investigation categories and their relevant questions discussed during these interviews.

\begin{tabular}{|c|l|}
\hline Section: & Example Questions \\
\hline A. Situational information & What are the outbreak criteria. \\
\hline B. Actors & $\begin{array}{l}\text { Identifying the organisations } \\
\text { involved. }\end{array}$ \\
\hline C. Processes & $\begin{array}{l}\text { Input feeds for decision support } \\
\text { systems. }\end{array}$ \\
\hline D. Determinants & $\begin{array}{l}\text { Determinants of successful } \\
\text { coordination. }\end{array}$ \\
\hline E. Resource management & Resource allocation efficiency. \\
\hline
\end{tabular}

In this paper, we discuss sections B and C (actors and processes) compiled in the form of inbound and outbound communication protocols. 


\section{Results}

We discuss outbound and inbound communication related to the H1N109 coordination dynamics separately. Inbound communication represents a node receiving communication, and outbound one represents a node establishing the communication channel. Such a link indicates the presence of a coordination relationship between both organisations.

\subsection{Outbound communication}

Outbound communication is discussed in two parts: formal and informal outbound communication.

\subsubsection{Formal outbound communication}

HSFAC team elicited the existing communication channels. It examined them, strengthened those which needs further support, and worked to bridge any structural holes. The communication plan displayed in figure 1 is named the "Pheromone communication", followed one-to-many, short and rapid. It represents the communication links starting from the state public health and ending at the 37 EDs in HNE hospitals. These links are used to distribute case definition as a one-page communication that enables unified cases admitted to the patients system.

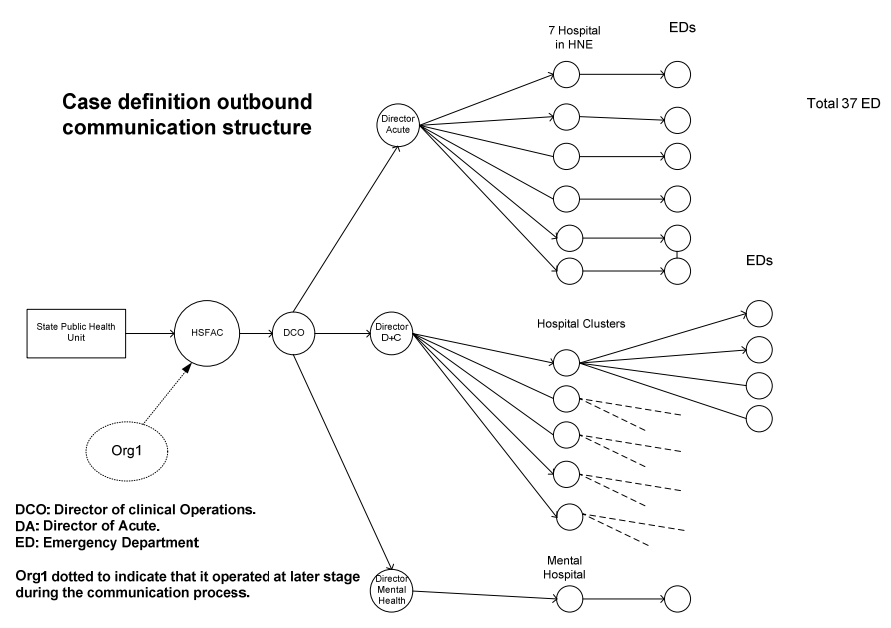

Figure 1: Outbound communication.

Figure 1 shows that HSAFC has strategically positioned itself on the path of communication that bridges the state public health and the director of clinical operations (DCO). By covering this structural hole, they were able to control and filter the information flow between the two nodes to the benefit of the ED nodes. The dissemination of the case definition is reduced from four hours to thirty minutes including the acknowledgment. It can be noticed that HSFAC 
were not positioned in a high degree centrality so not to be overburdened with communications overhead. They actually elicited the DCO's high degree centrality and its existing communication channels to pass the intended communication.

Emergent coordination would not be complete if it is not tested against interference and re-organisation. Eight weeks into the endemic an organisation, we will call it Org1, decided to activate its own communication protocols and started to disseminate large documents about policies and procedures to only part of the front line nodes (EDs) directly. This not only created message inconsistency, but also got the EDs to commit resources to different communication channels as well as to process and filter those communications. This situation was corrected by re-organising the communication structure by directing Org1 to communicate with the HSFAC directly which acted to bridge the structural hole again between the three networks.

\subsubsection{Informal outbound communication}

HASFAC team consciously used informal communication. This form of communication was found effective especially when they had to communicate with other bodies (medical and non-medical) outside their own jurisdiction. They extended to establish informal communication with the following parties:

(a) GPs: HNE has five divisions of general practitioners (GPs). Being the first point of contact for many potential cases and being geographically dispersed, the GPs represent an excellent network of information collection and an effective medium for message dissemination. This relationship was also used to train the GPs to build up their surge capacity to receive more patients rather than directing them to the hospitals and risking over-stretching of the respective EDs.

(b) Industrial Grid: HSFAC wanted to avoid industrial disputes that might arise from changing working conditions, and so updated the local business network about the outbreak and disseminated information on how to protect their workforce.

(c) Consultancy grid: In order to get advice from the required disciplines, the HSFAC co-opted expertise personnel from different domains: respiratory, clinical care, infection control, pharmacy and secondary workforce liaison officer.

(d) Ambulance Grid: Area Ambulance Services New South Wales (ASNSW) was not being updated about the latest outbreak information. An informal link was established to provide them with the latest updates.

One can deduce from the above information that some of the important features of the emerging coordination was successfully implemented by the HSFAC team:

- Active discovery of the structural holes and bridge building.

- Active development of the informal networks when needed.

\subsection{Inbound communication}

An important part of disaster management is the collation of data relating to damage impact. There will be many agencies gathering information each 


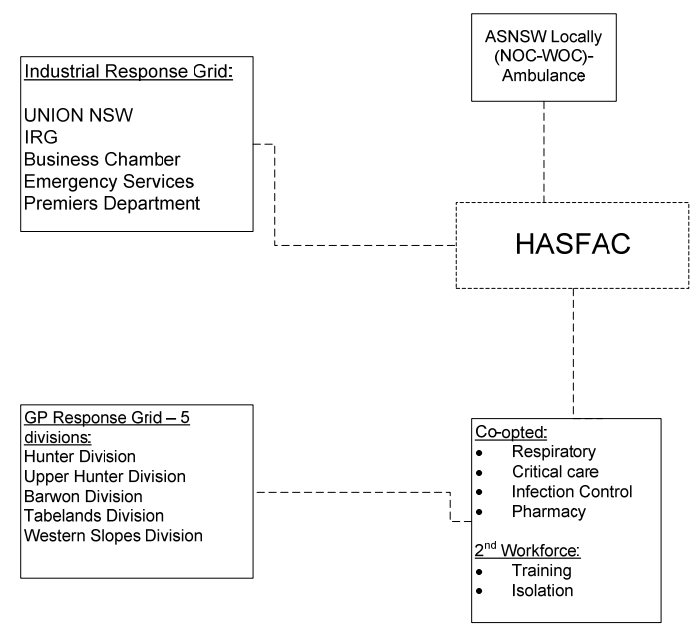

Figure 2: Informal outbound communication.

following its own protocols under its own jurisdiction. The coordination of the information collection effort and optimal investment of this information complete the data collection circle. Disease monitoring systems must be adaptive according to different criteria:

1- Case accuracy: Aims at minimising false positives which lead to an unnecessarily high case load, or false negatives which results in cases slipping the net.

2- Timely reporting of cases: Adding the cases to the case monitoring systems as soon as they are diagnosed for follow-up and monitoring.

3- Protecting resources: Intensive care units (ICU's) for example usually have a limited number of beds. Resources such as these need a "lead monitor" that will quickly flag the number of cases that are using or expected to use this resource.

4- The monitoring system needs to be distributed geographically and functionally in order to capture cases at early signs of development.

Based on these criteria, HSFAC established an inbound monitoring system to capture cases details with lead indicators on specifically critical resources.

Figure 3 below shows the network positions and functionality of each monitor. Again, the HSFAC did not position itself as the central node in the network. They aggregated the lag monitors, to the Public Health Emergency Operation Centre (PHEOC). On the other hand lead monitors, which have high urgency, were directly connected to the HSFAC in order to communicate their existence to relevant parties. Hence, HSFAC sacrificed the higher degree of centrality and betweenness, which would have meant a connection to every single node (star network). 


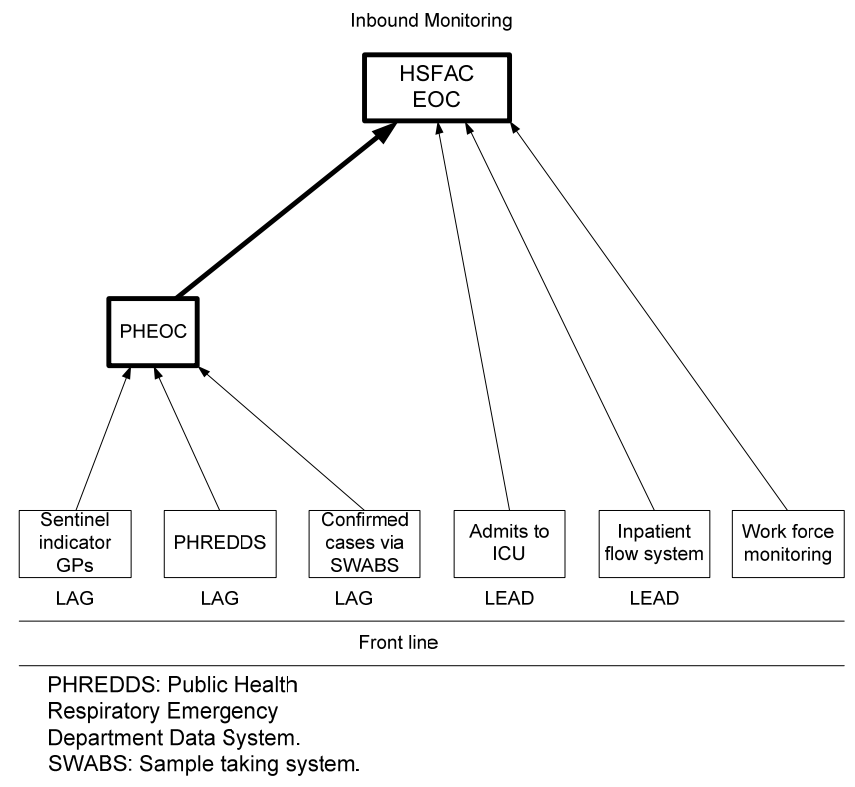

Figure 3: Lower level inbound communication.

\section{Conclusions}

The coordination of interventions for disease outbreaks is a complex task and, at the same time, is under-researched. A good coordination structure is expected to lower infection rate. In this exploratory study, we show the potential of using social network theory to analyse the communication channels implicated in the management of outbreaks. We illustrate this potential using a particular example of the management of an outbreak of H1N1 by the Hunter New England Area Health Service (HNEAHS). A qualitative analysis using network methods to address the complexity of the task, suggests design elements that optimise the level of resource use. In particular sacrificing the inbound and outbound high degree of centrality by using link aggregations might be feasible approach for demanding information dissemination organisations.

It is also important to reflect on the data collection approach that we followed for this study. The investigative approach provided us with the first "insight" into this domain. Further insight is required into the complexity and dynamics of these processes. Some of this information we intend to gather will be:

1. What type of agencies being involved at different stages of the outbreak phases?

2. How do the formal and informal relationships evolve during the coordination lifetime? 
3. What are the dynamics that affect nodes (organisations) and links performance during the outbreak?

We are planning to arrange follow up interviews with the players at key positions during the outbreak management and intervention period. We also aim to collect quantitative data that will be used for statistical validation leading to generalisations of our hypotheses generated by this study. Such data will provide a good foundation for structural and statistical analysis of the network. Combining qualitative and quantitative data with network analysis techniques, will provide a more powerful understanding of the coordination in outbreaks and creates an opportunity to propose an efficient disease outbreak coordination model based on network theory.

\section{References}

[1] Comfort, LK, Sungu, Y, Johnson, D, Dunn, M: Complex Systems in Crisis: Anticipation and Resilience in Dynamic Environments. Journal of Contingencies and Crisis Management, 9(3):144-158.2001

[2] Neal, DM, Phillips, BD: Effective Emergency Management: Reconsidering the Bureaucratic Approach. Disasters, 19(4):327-337.1995

[3] Chen, R, Sharman, R, Rao, HR, Upadhyaya, SJ: Coordination in emergency response management. Commun ACM, 51(5):66-73.2008

[4] Fraser, C, Riley, S, Anderson, RM, Ferguson, NM: Factors that make an infectious disease outbreak controllable. Proceedings of the National Academy of Sciences of the United States of America, 101(16):61466151.2004

[5] Newman, MEJ: Spread of epidemic disease on networks. Physical Review E, 66(1):016128.2002

[6] Eastwood, K, Durrheim, D, Massey, P, Kewley, C, Massey, K: Australia's pandemic 'Protect'strategy: the tension between prevention and patient management. Rural and Remote Health, 9:1288.2009

[7] Chandler: Strategy and structure: MIT press Cambridge, MA; 1973.

[8] Faraj, S, Xiao, Y: Coordination in Fast-Response Organizations. Management Science, 52(8):1155-1169.2006

[9] Malone, TW, Crowston, K: What is coordination theory and how can it help design cooperative work systems? In: Proceedings of the 1990 ACM conference on Computer-supported cooperative work. Los Angeles, California, United States: ACM; 357-370.1990

[10] N, K: Interorganizational coordination in dynamic context: networks in emergency response management. Connections, 26:35.2005

[11] Szabolcs Feczak, LH: Measuring Coordination Gaps of Open Source Groups through Social Networks. In: ICEIS 2009; 84-90.2009

[12] Miller, JH, Moser, S: Communication and coordination. Complexity, 9(5):31-40.2004 
[13] Iannella, R, Henricksen, K: Managing Information in the Disaster Coordination Centre: Lessons and Opportunities. In: May 2007 2007: Citeseer.2007

[14] De Wolf, T, Holvoet, T: Emergence Versus Self-Organisation: Different Concepts but Promising When Combined. In: Engineering Self-Organising Systems. Edited by Brueckner SA et al, vol. 3464: Springer Berlin / Heidelberg; 1-15.2005

[15] Hossain, L: Effect of organisational position and network centrality on project coordination. International Journal of Project Management, 27(7):680-689.2009

[16] Freeman, LC: Centrality in social networks conceptual clarification. Social Networks, 1(3):215-239.1978

[17] Granovetter, M: The Strength of Weak Ties: A Network Theory Revisited. Sociological Theory, 1(ArticleType: primary_article / Full publication date: 1983 / Copyright (C) 1983 John Wiley \& Sons):201-233.1983

[18] Hansen, M: The search-transfer problem: the role of weak ties in sharing knowledge across organization subunits. Administrative Science Quarterly, 44(1):82-85.1999

[19] Han, P: The informal organization you've got to live with. Supervisory management, 10(28):25-28.1983

[20] Cross, ROB, Borgatti, SP, Parker, A, Prusak, L: 6. Making Invisible Work Visible: Using Social Network Analysis to Support Strategic Collaboration. Creating Value with Knowledge, 1:82-103.2004 